Méchoulan, Éric, « La culture de la valeur. Économie et esthétique chez Kant et Schiller ", dans Jean-François Vallée, Jean Klucinskas et Gilles Dupuis (dir.), Transmédiations. Traversées culturelles de la modernité tardive, Montréal, Les Presses de l'Université de Montréal, 2012, p. 150-171. 


\title{
La culture de la valeur. Économie et esthétique chez Kant et Schiller
}

\author{
Éric Méchoulan
}

\begin{abstract}
La minutie, louable sans doute, avec laquelle on rédige à présent l'histoire contemporaine, doit malgré tout faire naître naturellement en chacun une inquiétude: celle de savoir comment nos descendants éloignés s'y prendront pour soulever le fardeau de l'histoire que nous pourrons leur laisser d'ici quelques siècles.
\end{abstract}

KANT, Idée d'une histoire universelle au point de vue cosmopolitique

\begin{abstract}
C
omme le souligne Françoise Proust, «est beau non pas un objet, non pas même ce qui suscite un sentiment de plaisir, mais ce qui apparaît à l'occasion de l'éveil d'un tel sentiment [...] : l'arrivée dans le temps ou avec le temps d'un apparaittre, le temps de l'apparaître ou l'apparaître du temps ${ }^{1} »$. Les jeux d'apparence de la grâce sociale reviennent ainsi dans une rhétorique du temps où la culture reconduit les ordres entrecroisés du sensible et de l'intelligible, du naturel et du sociable, de l'obligation et de la liberté. Sous le geste aristocratique du détachement et du plaisir mondain à paraître, on peut reconnaître les habits paradoxaux de l'esthétique. Ce n'est donc pas un hasard de voir revenir la faveur au cœur du désintérêt esthétique. Dès le premier moment (celui de la qualité), Kant ramasse en une formule frappante les étrangetés du goût qui le différencie des sensations de l'agréable et de la raison du bien (puisque l'homme sensible au beau partage l'animalité des sensations et le caractère raisonnable du bien): seul le goût suscite

[...] une satisfaction désintéressée et libre; en effet aucun intérêt, ni des sens, ni de la raison, ne contraint l'assentiment. [...] La faveur est l'unique satisfaction libre. Un objet de l'inclination ou un objet qu'une loi de la raison nous impose de désirer ne nous laissent aucune liberté d'en faire pour nous un objet de
\end{abstract}

1. Françoise Proust, Kant, le ton de l'histoire, Paris, Payot, 1991, p. 31. 
plaisir. Tout intérêt présuppose un besoin ou en produit un, et comme principe déterminant de l'assentiment, il ne laisse plus le jugement sur l'objet être libre ${ }^{2}$.

Le désintérêt ne sort pas l'individu des circuits sociaux, il ne le suppose pas pris seulement dans le filet des besoins, ni exactement dans le désir d'échanger des surplus, mais dans la grâce de sa liberté. Die Gunst, c'est la faveur, les bonnes grâces, c'est aussi le moment opportun, la bonne occasion (die Gunst des Augenblicks). Avec le jugement de goût, le plaisir naît de l'expérience où le temps est liberté.

On oublie, en effet, parfois ce lien nécessaire entre le désintérêt et la liberté, la liberté et la grâce, la grâce et le plaisir³. Loin des aplatissements sociologiques qui cherchent sous le désintéressement la simple dénégation d'un rapport social, il s'agit de saisir comment la réarticulation, chez Kant, de problèmes propres à l'histoire de la philosophie et de questionnements que traduisent gestes, postures ou styles des formations sociales, trouve une logique singulière et un sens du commun, une grandeur évidente et une exemplarité significative ${ }^{4}$. Le désintérêt ne recouvre donc pas seulement l'indifférence bourgeoise ou la morgue nobiliaire: il retraduit dans le vocabulaire d'une économie politique moderne le style du loisir mondain et de la grâce sociale. C'est pourquoi le grand moment du sublime, pour Kant, est moins l'enthousiasme que l'absence d'affections qui s'accorde avec la raison pure et dont il ne peut exprimer autrement la qualité qu'en recourant à la présentation d'un style aristocratique: «Un tel état d'âme se dit seul noble [edel]; ce terme est appliqué de manière dérivée aux choses, par exemple des édifices, un vêtement, une manière d'écrire, un certain maintien du corps, etc., lorsque celles-ci suscitent moins l'étonnement [...] que l'admiration [...]; et c'est là ce qui arrive quand des Idées dans leur présentation s'accordent sans intention et sans art en vue d'une satisfaction esthétique ${ }^{5}$.»

2. Emmanuel Kant, Critique de la faculté de juger, trad. par Jean Hyppolite, Paris, Vrin, $1981, \S 5$.

3. "Désintéressement, ce mot ne saura que prêter à malentendu et confusion tant qu'il ne sera pas lu dans sa connexion avec la liberté et la faveur.» Jérôme de Gramont, Kant et la question de l'affectivité. Lecture de la troisième critique, Paris, Vrin, 1996, p. 173.

4. Sur ce point, Pierre Bourdieu a beau affirmer - avec un usage de la distance et une revendication tacite de profondeur qui caractérisent justement l'esthétique qu'il malmène - «que la lecture naïvement réductrice, qui ramènerait le texte de Kant au rapport social qui s'y dissimule et s'y transfigure, ne serait pas moins fausse que la lecture ordinaire, qui le réduirait à la vérité phénoménale dans laquelle il ne se dévoile qu'en se voilant ", il n'est pas sûr qu'il ne cède pas - facilement? - à la satisfaction «de manifester les mécanismes sociaux qui conduisent à la dénégation des principes véritables [je souligne] du jugement de goût (et à leur re-dénégation dans toutes les lectures conformes [conformes selon le goût, ou plutôt le dégoût, du sociologue ?]) ", La distinction. Critique sociale du jugement, Paris, Minuit, 1979, p. 578.

5. Emmanuel Kant, Critique de la faculté de juger, «Remarque générale sur l'exposition des jugements esthétiques réfléchissants», p. 109. 
Les paradoxes esthétiques prolongent certains paradoxes de la société d'Ancien Régime: ainsi la valeur sociale allouée au travail double-t-elle celle que l'on accorde encore au loisir, à condition qu'ici et là, la liberté en demeure l'enjeu propre. La noblesse a perdu ses lettres de créance, pourtant l'aristocratie demeure un modèle d'existence: «Pendant deux siècles au moins la conscience du progrès bourgeois s'est faite dans la vie sociale française non seulement par l'accession à la noblesse, mais par un besoin jaloux de vivre selon un style noble ${ }^{6}$.» Mais ce style noble est, en fait, déjà la marque spécifique d'une aristocratie qui découvre son relief mondain dans une culture suscitée en partie par une bourgeoisie ou une noblesse financière et éduquée. Il ne s'agit pas de revenir au rôle dépassé du noble inculte et incivil (la brutalité de certains libertins témoigne, au contraire, d'un sens des raffinements de l'existence, même s'il s'écrit entre les lignes du sens commun).

Ce modèle très français trouve des échos de l'autre côté du Rhin. En dépit d'une opposition plus sourde et plus vive entre l'univers curial ou nobiliaire et la bourgeoisie intellectuelle, les Allemands récupèrent l'ordre aristocratique de la civilité et de la politesse en marquant une différence entre deux termes voués à connaître un long héritage: la civilisation et la culture ${ }^{7}$. Kant, là encore, noue de façon synthétique les problèmes:

Nous sommes hautement cultivés dans le domaine de l'art et de la science. Nous sommes civilisés au point d'en être accablés, pour ce qui est de l'urbanité et des bienséances sociales de tout ordre. Mais quant à nous considérer comme déjà moralisés, il s'en faut encore de beaucoup. Car l'idée de la moralité appartient encore à la culture; par contre, l'application de cette idée, qui aboutit seulement à une apparence de moralité dans l'honneur et la bienséance extérieure, constitue simplement la civilisation. Mais aussi longtemps que des États consacreront toutes leurs forces à des vues d'expansion chimériques et violentes, et entraveront ainsi sans cesse le lent effort de formation intérieure de la pensée chez leurs citoyens, les privant même de tout secours dans la réalisation de cette fin, on ne peut escompter aucun résultat de ce genre; car un long travail intérieur est nécessaire de la part de chaque communauté pour former à cet égard ses citoyens ${ }^{8}$.

6. Alphonse Dupront, Qu'est-ce que les Lumières?, Paris, Gallimard, 1996, p. 37.

7. Sur ces concepts dans le contexte allemand et sur les positions sociales des intellectuels comme Kant, voir Norbert Elias, La civilisation des moeurs (Paris, Calmann-Lévy, 1973, p. 11-51) et Henri Brunschwig, Société et romantisme en Prusse au XVIII siècle: La crise de l'État prussien à la fin $d u X_{V I I I}$ e siècle et la genèse de la mentalité romantique (Paris, Flammarion, 1973, p. 203-259).

8. Emmanuel Kant, «Idée d'une histoire universelle au point de vue cosmopolitique», $L a$ philosophie de l'histoire (opuscules), trad. par Stéphane Piobetta, Paris, Denoël, 1986 [1784], p. 39. Pour une étude détaillée de ce texte dans l'ensemble des écrits politiques de Kant, voir Gérard Raulet, Kant. Histoire et citoyenneté, Paris, PUF, 1996, p. 21-58. 
La civilisation ne serait donc que le vernis des bonnes manières posé sur le travail secret de la moralisation intérieure. Mais cela ne signifie pas qu'il faudrait que la première disparût pour faire place à la seconde. Si l'idée de moralité relève de la culture, son application constitue la civilisation. Plus importante est l'opposition entre civilisation-culture et guerre: la formation intérieure des êtres ne se joue pas simplement dans le travail privé que l'on produit sur soi; elle requiert une aide des États (au moins par la paix matérielle qui y serait assurée) et une fabrication intérieure de la communauté elle-même.

Le propre de la culture est d'appeler à un travail interne de chacun dans le travail général de la communauté: la moralisation est intersubjective ou elle n'est pas. En même temps que la société se donne une forme comme culture, les citoyens se fabriquent leurs personnages comme moraux: c'est là la marque du monopole bourgeois sur la moralité, en retrait de l'autorité naturelle de la noblesse, dans un pays où il est rare que les roturiers n'aient pas dans leur famille un pasteur 9 . Culture et morale forment les deux socles des positions publiques de la bourgeoisie intellectuelle, générant un modèle plus large où le destin de l'État et de la communauté doit tourner autour de ces deux pôles. À l'instar de ce que pensait Adam Smith, les individus agissent alors en fonction de leurs intérêts privés, mais ces intérêts sont immédiatement sociaux. La civilisation affiche une moralité des comportements que la culture fabrique secrètement.

La première occurrence connue, en France, du terme de civilisation souscrit aux mêmes enjeux. Elle se trouve dans un manuscrit de Mirabeau et pose déjà le problème en recourant à cette opposition et à ce lien entre l'apparence publique et la vertu intérieure:

L'homme est un animal sociable et ce n'est que comme tel qu'il peut être vertueux. [...] La reconnoissance est un dérivé de la connoissance: il faut, pour sentir ce que nous devons à Dieu, sçavoir ce que Dieu nous a donné. Et tous les dons dont il joncha notre passage ne se font sentir à nous que par la société. [...] ce n'est qu'envers nos semblables [...], ce n'est qu'avec eux et pour eux que nous pouvons être vertueux. [...] L'homme ne peut ètre heureux que par la vertu; il ne peut ètre vertueux que par la societé; tout ce qui [perd?] la societé décombre la vertu; la civilisation est l'amendement de la societé. [...]

Si je demandais à la plupart en quoy faites-vous consister la civilisation, on me repondroit, la civilisation d'un peuple est l'adoucissement de ses mours, l'urbanité, la politesse et les connoissances repandues de maniere que les bienseances y soient observées et y tiennent lieu de loix de détail, tout cela ne me presente que le masque de la vertu et non son visage, et la civilisation ne fait rien pour la societé si elle ne luy donne le fonds et la forme de la vertu. [...]

9. Voir Henri Brunschwig, Société et romantisme en Prusse au XVIII siècle, p. 206. 
L'homme reçut dans son origine l'empreinte de la loy de nature [...]. Ce sont les fausses notions et les préjugés qui offensent cette loy divine, préjugés d'invention ou de dépendance de la nature qui font la barbarie, et non l'ignorance de tel ou tel art, de telle ou telle autre découverte [...]; mais je vous avertis que j'ay toujours eu beaucoup d'esprit si au moins on peut parler ainsi, beaucoup d'idées; or on a abusé de mon esprit, et pourquoy cela, parce que je me suis avisé de vivre en bonne compagnie, c'est-à-dire, avec de fort honnestes gens par le cœur, et fort civilisés par l'esprit. [...] Ces honnestes gens gardent leur honneteté et leur cœur pour leur conduite, et leur civilisation et leur esprit pour la societé ${ }^{10}$.

Les dons originels de Dieu sont encore présents à l'horizon des êtres, mais la médiation de la société seule permet d'y avoir accès - qu'il s'éloigne encore un peu et l'ordre social demeurera seul en piste. Cette société, pour ce grand physiocrate qu'est Mirabeau, ne produit de la vertu qu'à raison de la reconnaissance des grâces divines: comme la nature est gracieusement fertile, la nature humaine est généreusement rentable, à condition d'en évaluer les nécessités communes. Nul n'est vertueux seul; réciproquement, la société a besoin de ses membres pour fabriquer la morale de tous. Cette fabrication sociale a pour nom civilisation. Elle peut prendre le masque de la politesse, elle devrait composer le visage de la vertu. Voilà pour la critique interne de son développement. La critique externe fait se retourner la civilisation contre la barbarie. Les lois premières, les lois divines ont été faussées par les préjugés; il s’agit de retrouver le sol originel. La civilisation permet de remonter le temps dès lors qu'elle ne consiste pas seulement en de provisoires inventions. La critique des préjugés cherche à dépouiller la culture de la barbarie en l'homme. Mirabeau se situe encore au point de jonction de deux conduites: l'une qui trouve dans l'origine - hélas pervertie - la source de la civilisation morale de l'homme; l'autre qui cherche dans la fabrication sociale de soi la tâche par où les êtres humains se réaliseraient enfin. Dans l'économie des individus, ces deux conduites ont leurs ancrages: ici le cœur avec l'honnêteté, là l'esprit avec la civilisation.

La culture est, pour Kant, ce qui sauve l'homme de la civilisation, comme l'opinion publique peut résister aux faux attraits de la politesse nobiliaire. Il faut loger le loisir aristocratique au cœur du travail social sur soi, la légèreté de la grâce et de la faveur dans la liberté laborieusement bâtie. Car Kant ne se contente pas de diviser l'homme entre son cour et sa raison. Tout son propos consiste au contraire à mettre en scène les antinomies et à découvrir à la fois leurs ancrages nécessaires et leurs illusions régulatrices. Il résume ainsi tout le mouvement de civilisation des êtres dans la puissance

10. Mirabeau, L'amy des femmes ou traité de la civilisation, c. 1756, Archives nationales, $\mathrm{M} 780 \mathrm{n}^{\circ} 3$. Je souligne. 
d'une antithèse: c'est l'insociable sociabilité des hommes qui, à la fois, les pousse à s'associer et les fait résister à ce rassemblement. La sociabilité développe leurs dispositions naturelles; l'insociabilité les engage à surmonter leur paresse tout aussi naturelle pour se frayer une place parmi leurs compagnons sous l'impulsion du désir de domination ou de cupidité. «L'homme a alors parcouru les premiers pas, qui de la grossièreté [aus der Rohigkeit] le mènent à la culture [zu Kultur] dont le fondement véritable est la valeur sociale de l'homme; c'est alors que se développent peu à peu tous les talents, que se forme le goût» et même que se transforment les quelques dispositions des hommes au discernement moral en des "principes pratiques déterminés ${ }^{11} »$.

Il ne s'agit donc pas d'aller pieusement aux sources heureuses de la bonté originelle des hommes, mais de saisir l'indispensable et foncière ambivalence qui, simultanément, les pousse vers les autres hommes et les repousse de leurs compagnons. Technique, goût, morale sont l'effet de la lutte autant que de l'attrait; ils installent l'homme sur la scène de l'histoire. Que cette histoire soit celle d'un progrès est manifeste, mais un progrès lent, difficile, et certainement pas linéaire ou invincible. La téléologie donne une idée de finalité aux affaires humaines; elle ne passe jamais purement et simplement dans les faits.

La culture serait donc cette disposition acquise des êtres à produire de la valeur par l'antagonisme constant qui les anime. Les bergers d'Arcadie connaissent une existence de concorde et de paix où rien ne se passe, puisqu'ils ne lui donnent "guère plus de valeur que n'en a leur troupeau domestique ${ }^{12} »$ : ils se tiennent dans une paresse naturelle qui évite le besoin des autres autant qu'elle esquive le souci de soi. Pour que la culture produise de la valeur, elle doit entraîner l'homme dans le conflit - ou plutôt, c'est la nature qui pousse ainsi l'homme à se cultiver par le conflit: «L'homme veut la concorde, mais la nature sait mieux que lui ce qui est bon pour son espèce: elle veut la discorde. Il veut vivre commodément et à son aise; mais la nature veut qu'il soit obligé de sortir de son inertie et de sa satisfaction passive, de se jeter dans le travail et dans la peine pour trouver en retour les moyens de s'en libérer sagement ${ }^{13}$." Sous ce lexique volontariste ou naturaliste, il s'agit de reconnaître un déploiement qui est rien moins qu'automatique. À la différence des animaux auxquels la nature a distribué les instincts nécessaires pour leur existence, elle n'a donné à l'homme que la puissance de se fabriquer lui-même. La pure concorde ramènerait les

11. Emmanuel Kant, «Idée d'une histoire universelle au point de vue cosmopolitique», op. cit., p. 31-32.

12. Ibid., p. 32. Je souligne.

13. Ibid., p. 32. 
êtres humains à leur statut animal. La simple discorde les anéantirait. Le conflit des forces en présence fait surgir des valeurs là où ne passaient que des moyens de fuir ou des envies de se satisfaire. Le travail devient ainsi le maître mot des destinées sociales.

La finalité n'est rien d'autre qu'un retour au point de départ: sortis d'un repos indéfini et merveilleusement solitaire par la nécessité de l'ouvrage commun, les hommes ne travaillent vraiment que pour parvenir à s'en libérer et refonder en eux la sagesse du loisir infini. Le repos ne produit rien: ni valeur, ni véritable loisir, ni authentique liberté. Travail et culture sont indispensables pour que le loisir paraisse autre chose qu'une dissipation paresseuse des instants. En un retournement solidaire des tensions sociales et des paradoxes politiques contemporains, le travail doit apparaître comme la négation vitale du repos, mais le loisir doit être produit comme la finalité du travail ${ }^{14}$.

À la fabrication intérieure des êtres répond ainsi, comme un écho d'écho, le travail social de la culture; dans la communicabilité des voix s'établissent les valeurs. C'est pourquoi - si l'on reprend le chemin de la troisième Critique - l'intérêt du jugement esthétique existe bien dans la communauté des êtres, mais à la suite du jugement et dans l'empirique, non en son fondement et sur le plan transcendantal. Le raffinement des sensations ne suffit pas (on resterait alors dans l'agréable et dans le sentiment personnel) pour engendrer un jugement esthétique: il faut pousser la civilisation jusqu'à l'idée même de l'échange et découvrir dans le sentiment de plaisir qu'on éprouve «l'idée de sa communicabilité universelle [qui] en accroît presqu'infiniment la valeur ${ }^{15}$ ». La valeur est produite par l'échangeabilité; c'est celle-ci qui permet de s'extraire du préjugé personnel. Dans les faits, je peux avoir du mal à communiquer mon sentiment

14. Juxtaposons simplement à ce texte de Kant une doléance féminine, afin de saisir vite le fond commun du rapport au travail, à la culture et à la moralité humaine. Les femmes du Tiers-État semblent vouées à deux régimes: sans beauté et sans dot, elles végètent auprès de misérables artisans; «si, au contraire, elles naissent jolies, sans culture, sans principes, sans idée de morale, elles deviennent la proie du premier séducteur» («Pétition des femmes du Tiers-État au roi, 1er janvier 1789", Cahiers de doléances des femmes en 1789 et autres textes, préf. Paule-Marie Duhet, Paris, Éditions des femmes, 1981, p. 27) et chutent dès lors dans le libertinage de la marchandise. Cette lettre de doléance réclame alors, d'un côté, un monopole de certains emplois (couturière, brodeuse, marchande de modes), d'autre part, une condamnation visible de ces filles qui remettent en cause l'estime portée aux femmes honnêtes: "Il faudrait que, sous peine de travailler dans des atteliers [sic] publics, au profit des pauvres (on sait que le travail est la plus grande peine qu'on puisse leur infliger), elles ne puissent jamais quitter cette marque.» (p. 28) Faute de culture (donc de morale), les femmes sont séduites aisément, et devraient, pour réintégrer l'ordre social, passer du côté du travail: rejeté pour ces femmes sans culture, indispensable pour les femmes estimables. L'éducation morale, le goût de l'instruction, bref, la culture, s'oppose aux séductions immédiates du loisir et de la débauche. Culture et travail coulent de source: une même source.

15. Emmanuel Kant, Critique de la faculté de juger, $\$ 41$. 
de plaisir, il peut n'être jamais partagé; de droit, je le sens partageable. Voilà pourquoi le jugement esthétique opère comme un jugement déterminant (c'est-à-dire conceptuel) en réclamant de chacun un partage du même sentiment, sans, pourtant, user de détermination conceptuelle. Ce jugement est, pour Kant, réfléchissant. Dans la réflexion qu'il engendre en moi, je sens la densité inattendue de mon propre sentiment, gonflé qu'il est de la présence universelle d'autrui. Puisqu'il n'y a pas de détermination, par avance, ce jugement repose sur l'harmonie contingente de l'imagination, en sa liberté, et de la légalité de l'entendement en général (autrement dit, non de telle ou telle loi qui déterminerait un objet, mais de la faculté des règles dans son mouvement propre) : c'est une «légalité sans $l o i^{16}{ }$. En deçà donc d'une loi déterminante, d'une médiation, le jugement réfléchissant met en mouvement, immédiatement, le «fait transcendantal» qu'il y a de la loi: le jugement de goût «lie immédiatement la satisfaction ou la non-satisfaction à la simple contemplation de l'objet, sans en considérer l'usage ou la fin ${ }^{17}$ ».

La valeur ne provient ni de l'usage que l'on peut faire de l'objet, ni de l'échange dans lequel pourrait entrer la marchandise, mais de l'usage personnel de l'objet dans le sentiment que l'on éprouve et de l'échangeabilité de ce sentiment que l'on ressent de façon nécessaire. Le sentiment du beau engage ainsi l'imagination à produire librement de la légalité, mettant à jour un accord inespéré avec la faculté des concepts. Dans le sentiment du sublime, c'est avec la raison que l'imagination est censée s'accorder et l'homme a besoin de la culture, encore plus que pour le beau, afin d'éprouver dans le désaccord même entre les facultés une poussée bénéfique: «Sans développement des idées éthiques [sittlicher Ideen], ce que, préparés par la culture, nous nommons sublime ne paraîtra qu'effrayant à l'homme inculte [dem rohen Menschen, l'homme brut, comme on parle d'un matériau brut $]^{18}$.» Il faut la culture pour y adosser la finitude humaine et la préparer à prendre le point de vue de Dieu.

Le jugement réfléchissant suppose, en effet, dans ce geste qui replie le sentiment sur lui-même, de pouvoir découvrir, au moins à l'état d'idée régulatrice (non déterminante a priori), le point de vue infini de la totalité.

16. Ibid., «Définition du beau tirée du quatrième moment», p. 81. «Si donc dans les jugements de goût l'imagination doit être considérée dans sa liberté, elle ne sera pas comprise comme reproductive, comme lorsqu'elle est soumise aux lois de l'association, mais comme productive et spontanée [produktiv und selbsttätig, littéralement: auto-agissante] (en tant que créatrice de formes arbitraires d'intuitions possibles); [...] on comprend néanmoins fort bien, que l'objet puisse justement lui fournir une forme comprenant une composition du divers telle que l'imagination, si elle était livrée à elle-même, en liberté, serait en état de l'esquisser en harmonie avec la légalité de l'entendement en général.» (p. 80)

17. Ibid., p. 81.

18. Ibid., $\$ 29$. 
Kant a montré le caractère parfaitement illusoire de l'idée de Dieu, mais sans renoncer à en faire jouer la nécessité régulatrice à l'horizon des actions humaines: sous ce point de vue, toute la matérialité sensible s'accorde à la forme intelligible ${ }^{19}$. En recentrant sur la finitude même du sujet humain la perspective, Kant permet justement de saisir l'idée de Dieu dans l'opération d'un comme si, et le beau ou le sublime peuvent désormais évoquer cet inaccessible accord. La culture apparaît alors comme l'inscription intersubjective de cette idée régulatrice de Dieu. Tout ce qui était donné intégralement par Dieu doit pouvoir être produit librement par les hommes, ne serait-ce que sous le couvert d'apparences civilisées et du libre jeu des facultés.

Cela ne signifie pas que tout se réduit à un trivial conventionnalisme ${ }^{20}$, car la nature humaine, et non la culture, fonde la disposition au sentiment pour les idées pratiques (le sentiment moral). Cependant, la culture fabrique leur dispositif. La finitude de l'homme est à la fois ce qui en détermine la vanité, et qui, de ce vide, ordonne le libre remplissage. L'homme est contraint par la nature à produire, selon son gré, de la culture. La culture n'est jamais un fondement, mais une source indéfinie que les hommes s'inventent pour eux-mêmes:

La nature a voulu que l'homme tire entièrement de lui-même tout ce qui dépasse l'agencement mécanique de son existence animale, et qu'il ne participe à aucune autre félicité ou perfection que celle qu'il s'est créée lui-même [...]. La nature semble même s'être ici complu à sa plus grande économie [...] comme si elle voulait que l'homme, en s'efforçant un jour de sortir de la plus primitive grossièreté pour s'élever à la technique la plus poussée, à la perfection intérieure de ses pensées, et (dans la mesure où c'est chose possible sur terre) par là jusqu'à la félicité, en doive porter absolument tout le mérite, et n'en être redevable qu'à lui-même $e^{21}$.

Hors de l'univers des dettes comme du monde des contraintes, l'être humain agit comme s'il était véritablement autonome, comme s'il se donnait à lui-même ses propres lois. Il ne figure, bien entendu, qu'un quasisouverain. Les dettes sont bien présentes, mais elles ne sont jamais présentées, sinon sous la forme d'une autodétermination du sens moral.

19. Sur ce caractère quasi leibnizien de l'idée de Dieu, voir Luc Ferry, Homo aestheticus. L'invention du goût à l'âge classique, Paris, Grasset, 1990, p. 120-121.

20. «Mais parce que le jugement sur le sublime de nature a besoin d'une certaine culture [...], il n'est pas toutefois pour cette raison primitivement produit par la culture et introduit comme quelque chose de seulement conventionnel dans la société; au contraire il possède son fondement dans la nature humaine.» Emmanuel Kant, Critique de la faculté de juger, $\$$ 29.

21. Emmanuel Kant, «Idée d'une histoire universelle au point de vue cosmopolitique», op. cit., p. 29-30. Je souligne. 
Chez Kant, la culture fonctionne, en fait, comme une ruse de la nature ou, si l'on veut, comme une grâce souveraine.

Entre l'agréable qui ne cultive pas et le bien qui passe par-delà toute culture, le beau, exemplaire des jugements réfléchissants, «nous cultive, en nous enseignant à prêter attention à la finalité dans le sentiment de plai$\operatorname{sir}^{22}$ » - la finalité, au sens téléologique du terme (comme la deuxième partie de la Critique de la faculté de juger le mettra de l'avant), mais non la fin qui suppose un intérêt. Le beau nous prépare à apprécier un objet de façon désintéressée, et le sublime, au besoin, contre notre intérêt: le jugement réfléchissant, quoique bien ancré en nous, s'élargit jusqu’au sens de la communauté.

\section{Le sensus communis et l'exemplarité}

Il faut écarter d'office le repli du sensus communis sur un consensus empirique. C'est l'«autonomie du sujet jugeant du sentiment de plaisir $^{23}$ » qui réclame une universalité de principe sans avoir, pourtant, de concepts pour en déterminer l'effectivité, ni avoir fait d'enquêtes statistiques pour savoir si tout le monde partage son avis. Les héritages du goût peuvent former le jugement, ils ne déterminent, ils ne plient jamais le simple plaisir esthétique, celui qui n'offre que son seul pli (Einfalt). La communauté des connaisseurs exerce, bien sûr, le jugement de chacun de façon éclairée; mais ce n'est pas tant la culture qui détermine le fond des jugements, que les jugements, dans leur autonomie propre, qui favorisent la sociabilité et ouvrent à la culture. Kant entend distinguer l'usage courant de «sens commun» (qui désigne "le vulgare, qui se rencontre partout et dont la possession n'est absolument pas un mérite ou un privilège [Vorzug]», ce vulgare étant par définition "encore inculte ${ }^{24}$ [nicht noch kultiviert]») du sensus communis qui est l'idée d'un sens commun à tous, autrement dit d'une faculté de juger par où, dans l'usage de sa réflexion, chacun rattache son jugement particulier à la représentation de tout autre homme.

Trois maximes du sensus communis sont importantes: 1) penser par soi-même (maxime de l'entendement qui rejette la passivité du préjugé dont les Lumières cherchent à se libérer); 2) penser en se mettant à la place de tout autre (maxime de la faculté de juger qui entend faire de la pensée un usage final [zweckmäßigen Gebrauch], sans qu'il soit question des facultés

22. Emmanuel Kant, Critique de la faculté de juger, «Remarque générale sur l'exposition des jugements réfléchissants», p. 104.

23. Ibid., $\$ 31$.

24. Ibid., $\$$ 40. Comme on le note aisément, le registre de la culture ou de l'inculture se déploie dans les termes des hiérarchies sociales du vulgaire et du privilégié. 
de connaissance (déterminantes), mais de la manière de penser, du style même de la pensée [Denkungsart]) ; 3) toujours penser en accord avec soimême (maxime de la raison qui s'appuie sur les deux précédentes). Pour Kant, ce serait même plutôt le goût que le bon sens qui devrait recevoir le titre de sensus communis, dans la mesure où le goût «rend notre sentiment, procédant d'une représentation donnée, universellement communicable sans la médiation d'un concept ${ }^{25} »$. Tout le travail de médiation que propose la culture se bâtit donc sur ce passage immédiat du sentiment particulier à la règle publique: la production des comparaisons, l'éducation esthétique sont simultanément exaltées et effacées dans ce passage du vulgaire au privilégié. L'usage de la réflexion, l'attention portée au style même du jugement dans le jugement réfléchissant permettent la valorisation d'une production gracieuse de soi et la disparition propice du travail de médiation sociale ${ }^{26}$.

Mais sur quoi édifier la légitimité de cette immédiateté? Comment assurer le passage du sentiment au jugement, sans qu'il soit pris d'office dans les filets de l'entendement ou dans les rets de la raison? La réponse de Kant est: l'exemplarité. "Comme nécessité, conçue dans un jugement esthétique, elle [la satisfaction] ne peut être appelée qu'exemplaire, c'est-àdire, c'est la nécessité de l'adhésion [Beistimmung, l'assentiment] de tous à un jugement, considéré comme un exemple d'une règle universelle que l'on ne peut énoncer ${ }^{27}$.» Dans le jugement esthétique, le sentiment réclame, par principe, l'idée indéterminée du suprasensible en chacun; mais si cette idée est indéterminée, elle ne saurait être à la source du jugement, elle en figure plutôt le repos: "L'exemple n'exemplifie pas une Idée, il ne vient pas après, il n'est pas construit d'après, mais il est son fait, sa position, son affirmation. On va de l'exemple à l'Idée, non comme on va du posant au posé, mais comme on va de la position à sa pose. L'exemple est bien exemplaire, mais d'une déclaration d'exemplaritée ${ }^{28}$." Le mécanisme de l'opinion publique, dans l'ordre sociologique, trouve ici sa traduction transcendantale, à condi-

25. Ibid., $\$ 40$.

26. Éliane Escoubas indique bien que, la nature se présentant dans le sublime comme simplicité (Einfalt, littéralement "plié une fois», \$29), "ce qui, à l'œuvre dans toute monstration, ne se montre pas soi-même est la forme - autre nom du style». Or, «le texte de Kant fait retour une seconde fois sur la thématique de la forme, comme style, comme façon et façonnement dans la dimension de l'Un-pli. Et de façon symptomatique, c'est dans le retour du texte sur lui-même». Au $\$ 49$, Kant remarque, en effet, que des deux façons d'exposer ses pensées, l'une est une manière (le modus ostheticus), l'autre est une méthode (le modus logicus). «Il est manifeste ici que la manière de la pensée ou de l'art, comme le style de la nature, est ce qui ne peut être assimilé à une détermination et ne fait donc pas l'objet d'une présentation, mais constitue le procès même de la présentation. " (Imago mundi, topologie de l'art, Paris, Galilée, 1986, p. 45-46.) Pour le dire autrement, le style de l'immédiateté se retourne en immédiateté du style.

27. Emmanuel Kant, Critique de la faculté de juger, $\$ 18$.

28. Françoise Proust, Kant, le ton de l'histoire, p. 120. 
tion d'y ajouter la rhétorique mémorielle de l'exemple. Chez Kant, on voit comment les problématiques inédites de la sphère publique et de la raison des Lumières s'appuient encore, au besoin, sur un usage en quelque sorte recyclé de la mémoire collective. Là où l'immédiateté du passé donnait à l'exemple une actualité intelligible, la présentation immédiate lui alloue une réflexion temporelle. La rhétorique mémorielle permettait de déployer toute une prudence politique, autrement dit, une intelligence du quotidien. La réflexion exemplaire réclame une sensibilité historique. "Avoir l'oreille historique, c'est donc être attentif et vigilant envers les cas exemplaires, savoir entendre l'Idée qui s'y donne [...]. Cela demande un esprit judicieux, casuistique et, sinon tacticien, du moins rusé et stratège ${ }^{29}$.» C'est sur cette qualité tonale que l'éducation peut porter.

La communauté de jugement ne se fait pas a priori et elle n'est pas reçue par héritage: elle ne fait l'objet ni d'un savoir déterminant ni d'un préjugé du passé, mais de la réflexion d'un sentiment dans la forme duquel apparaît une communauté de sensibilité, une communauté historique. L'assentiment (Bei-stimmung) plutôt que la volonté générale, tel est ce qui compose une communauté dans l'histoire: l'humeur d'une succession et non l'obligation d'une imitation. Kant reconnaît parfaitement que l'on puisse élire certains auteurs classiques «comme s'ils formaient une noblesse entre les écrivains, qui par son exemple donne au peuple des $\operatorname{lois}^{30}$ ", mais ce n'est pas une négation de l'autonomie du goût que de valider ces exemples a posteriori, car ils relèvent d'un juste sentiment de la succession et non d'une plate imitation. Or, le goût est justement le jugement (puisqu'il ne repose pas sur des concepts) qui «a le plus besoin des exemples de ce qui dans le développement de la culture a reçu le plus longtemps l'approbation, s'il ne veut pas redevenir grossier et retomber dans l'état inculte du premier essai ${ }^{31}$ ». Le passé n'impose pas sa marque inconditionnelle sur les productions présentes, il les inspire au besoin et il évite de repartir à zéro. Mais cela n'empêche en rien le goût de se développer dans le jeu de sa propre liberté, à partir de la tonalité de l'histoire qui sera à la fois formatrice et objet de sa sensibilité.

L'exemplarité à l'œuvre dans le jugement de goût ne repose pas sur le sujet volontaire de la politique ou de la connaissance, mais sur le sujet sentimental, affectif, de la reprise, de la ruse et de la résistance. Prolongement de la sympathie humienne ou smithienne, puisque la sympathie permet de

29. Ibid., p. 120.

30. Emmanuel Kant, Critique de la faculté de juger, $\$ 32$. Je souligne à la fois la procédure régulatrice du comme si et la valeur nobiliaire de l'exemplarité.

31. Ibid., $\$ 32$. 
penser et de sentir en se mettant à la place des autres ${ }^{32}$ ? Hume et Smith avaient bien perçu que cette sympathie ne pouvait être que partiale, donc partielle: certains êtres nous demeurent profondément antipathiques, d'autres nous sont immédiatement proches sans qu'on puisse toujours en rendre raison. C'est pourquoi Kant ne conçoit pas cette communauté comme reposant sur une sympathie généralisée, une sympathie impartiale: ce serait là une illusion transcendantale. Il la pense comme sentiment immédiat de l'exemplarité d'un événement: dans le mouvement même de l'exemplarité se tisse l'assentiment nécessaire qui en appelle à une communauté (qui ne la fonde pas pour autant). La culture est l'illusion transcendantale, la production sociale, de cette communauté. Par un côté, elle nous ouvre à la liberté; par un autre, elle nous réduit au sens commun.

Encore une fois, il ne s'agit pas de glisser paisiblement de la culture esthétique aux idées morales. Tout ce que le beau et le sublime permettent est une analogie de l'expérience: on peut y

rattacher une disposition de l'esprit [eine Stimmung des Gemüts, un sentiment de l'âme] qui est semblable [ähnlich] au sentiment moral et bien que l'immédiat [unmittelbar] plaisir pris à ce qui est beau en la nature présuppose et cultive [kultiviert] une certaine libéralité [Liberalität] dans la manière de penser [Denkungsart] [...], toutefois la liberté [Freiheit] est plutôt par là représentée [vorgestellt] dans le jeu que soumise à une activité conforme à la loi ${ }^{33}$.

L'impeccable circularité de la liberté et de la loi dans la raison pratique est simplement jouée ou rejouée dans l'abandon à l'imagination. Du style de la libéralité qui peut se cultiver, on peut sauter dans le miroir tendu par le sentiment moral de la liberté, mais de même que le plaisir est immédiat, l'analogie l'est aussi: elle ne fait jamais l'objet d'une représentation médiatisée par des concepts universels. La libéralité n'est donc pas l'équivalent univoque de la liberté, elle en représente le possible jeu entre imagination et raison (le fait même que Kant choisisse le mot d'origine latine Liberalität plutôt que le terme d'origine germanique Freigebigkeit qui reprend Freiheit, signale la distinction que l'on doit maintenir entre la libéralité et la liberté). La générosité et la grâce sont encore des manières de mobiliser la liberté

32. «La propédeutique pour tous les beaux-arts, lorsqu'il s'agit du suprême degré de leur perfection, ne semble pas consister dans des préceptes, mais dans la culture des facultés de l'âme grâce à ces connaissances préliminaires que l'on nomme: humaniora, sans doute parce que humanité signifie d'une part le sentiment universel de sympathie, d'autre part la faculté de pouvoir se communiquer d'une manière intime et universelle; ces qualités constituent la sociabilité convenant à l'espèce humaine.» Emmanuel Kant, Critique de la faculté de juger, «Appendice. De la méthodologie du goût», p. 177.

33. Ibid., "Remarque générale sur l'exposition des jugements esthétiques réfléchissants", p. 105-106. 
humaine, elles n'en constituent plus l'essence souveraine ${ }^{34}$, car elles participent d'une pensée de la production où l'héritage du passé demeure au mieux comme exemple (d'un génie pour un autre génie), au pire comme préjugé (qu'il faut démonter) et où tout le poids de la légitimation porte sur le futur: non seulement comme progrès indéfini (même avec les errances que cela peut encore supposer), mais surtout comme traces d'une téléologie. La seconde partie de la Critique de la faculté de juger porte bien sur la faculté de juger téléologique (dont les jugements esthétiques offrent une préfiguration) et sur la formation culturelle que cela concourt à développer : «Produire dans un être raisonnable l'aptitude générale aux fins qui lui plaisent (par conséquent dans sa liberté) est la culture. Ainsi seule la culture peut être la fin dernière que l'on a quelque raison d'attribuer à la nature par rapport à l'espèce humaine ${ }^{35}$.» La nature engage ainsi l'homme à produire, mais ce qu'il produit est dû à sa propre liberté, faisant de la culture un éloignement toujours plus évident de l'ordre naturel. La culture devient ainsi une discipline des manières où le maximum de liberté se conjoint au maximum de contrainte ${ }^{36}$.

\section{La grâce et la dignité : de Kant à Schiller}

Si Kant met en valeur la tension entre le travail et la grâce, la libéralité et la liberté, le particulier et l'universel, jusque dans les résistances qu'elle provoque, Schiller, qui entend bien le propos kantien, voudrait porter la

34. Autant par le rapport institué entre les particuliers et l'universel, entre les individus et l'État qu'entre la libéralité et la liberté, Kant participe d'une philosophie du libéralisme politique et économique qui est héritage à la fois de Hume et de Smith en même temps que résistance à leur empirisme. Voir sur ces points complexes, sur lesquels je ne peux m'engager ici, Anthony Cascardi, "Aesthetic Liberalism: Kant and the Ethics of Modernity", Revue internationale de philosophie, 1991, $\mathrm{n}^{\circ}$ 176, p. 10-23, et Lucien Jaume, La liberté et la loi. Les origines philosophiques du libéralisme, Paris, Fayard, 2000, p. 257-310.

35. Emmanuel Kant, Critique de la faculté de juger, $\$ 83$. Il est curieux que Jean-François Lyotard termine son ouvrage sur la politique kantienne en tâchant de conserver l'idéal d'une transformation des différends en litiges et des idiomes en argumentations «sans le secours de l'Idée que la nature poursuit dans l'histoire la fin de la liberté humaine, sans l'hypothèse téléologique» et que ses dernières phrases soient néanmoins: «Peut-être la responsabilité réflexive aujourd'hui est-elle aussi de discerner, de respecter et de faire respecter les différends [...]. Ce serait de la sorte être fidèle, sans paradoxe, à l'Idée kantienne de "culture" entendue comme trace de la liberté dans la réalité: elle est, écrit Kant, "l'aptitude à se proposer en général des fins".» (L'enthousiasme: La critique kantienne de l'histoire, Paris, Galilée, 1986, p. 114.) Sauf à voir dans l'hypothèse téléologique une détermination a priori et non une perspective régulatrice, il est difficile de comprendre son rejet. Pour Kant, comme l'entend Lyotard dans ses derniers mots, la téléologie n'est nécessaire que pour autant qu'elle témoigne d'une aptitude à se proposer des fins: il n'y a pas de fin déterminée, pas de raison ou de sens de l'histoire, mais il y a de la raison et du sens dans les productions historiques (ce pourquoi, empiriquement, nous pouvons en déchiffrer des modèles et en proposer des interprétations intelligibles pour le passé et orienter nos actions pour le futur).

36. Voir Emmanuel Kant, «Idée d'une histoire universelle au point de vue cosmopolitique», p. 33-34. 
tension jusqu'à son point de résolution musicale ${ }^{37}$. De la grâce et de la dignité est écrit par Schiller à la suite de sa lecture attentive de la troisième Critique. La grâce est, à ses yeux, comme la ceinture de Vénus qui peut s'ajouter à la beauté ou à la noblesse et sans laquelle celles-ci ne séduisent pas vraiment (même ce qui n'est pas beau peut apparaître comme gracieux). Beauté mobile, voire beauté du mouvement, la grâce est à la fois objective, puisqu'elle appartient à l'objet, et contingente, puisqu'elle peut s'en détacher. Cette contingence ne saurait passer pour une limitation: le temps lui-même (dans le mouvement ou dans l'occasionnel) fait la séduction de la grâce. Là où la beauté est donnée, la grâce est la beauté de mouvements contingents qui n'impliquent pas un rétrécissement du nécessaire au hasardeux, mais au contraire qui supposent un élargissement du concept de beau: le temps est un surcroît, non un déclin. On conçoit alors que la grâce soit produite par le sujet lui-même et non reçue de la nature et qu'elle participe, dès lors, de la culture entendue au sens de Bildung (formation) et non simplement de Kultur.

Pour Schiller, la liberté gouverne la beauté: que ce soit la beauté de la construction pour la nature ou la beauté du jeu pour l'âme humaine. Cette beauté architectonique fait honneur au Créateur, mais la grâce fait honneur à son propriétaire, puisqu'elle est l'expression de sa personne (rejoignant ainsi les conceptions des droits des auteurs). Le génie, quoiqu'il relève de la personne, vaut en ce qu'il passe pour naturel, «et à cause des préjugés qu'ont les hommes, on louera davantage ce qui s'obtient sans peine et sans mérite. De même la beauté sera plus admirée que le charme et le génie que la puissance acquise de l'esprit. C'est par leurs travers [...] que ces deux enfants gâtés de la nature se font remarquer, comme s'ils étaient de haute naissance ou qu'ils appartenaient à quelque caste élevée ${ }^{38} »$. On sent bien la méfiance du roturier envers les illusoires productions naturelles de la noblesse: la grâce ne se résume pas à un don, une gratuité, un effacement de tout ce qui en fait une marchandise échangée à l'étal du beau monde. Le génie devrait «se fortifier grâce aux principes, au goût et à la science

37. "Nous avons évoqué en passant l'idée de Herder qui présente l'ouïe, la sphère acoustique, comme le centre vivant de la Besonnenheit, de ce trait qui pénètre et prédétermine la nature à la fois spirituelle et sensible de l'homme. Chez Schiller, cette idée est associée à la théorie kantienne du temps en tant que forme du sens interne et milieu universel que l'imagination créatrice utilise à des fins de schématisation, pour faire pénétrer les principes purement idéels dans la sphère du sensible.» Jan Patocka, L'art et le temps, trad. par Érika Abrams, Paris, Presses Pocket, 1990 [1971], p. 164 et 169-17o. Pour une comparaison rapide, voir Dieter Henrich, «Beauty and Freedom: Schiller's Struggle with Kant's Aesthetics», Essays in Kant's Aesthetics, Ted Cohen et Paul Guyer (dir.), Chicago, University of Chicago Press, 1982 , p. $237-257$.

38. Friedrich Schiller, De la grâce et de la dignité, trad. par Constance Chastenet, Paris, Hermann, 1998, p. 71, n. 5. 
[...], il devrait, au moment opportun, penser à s'assurer de ce don ambigu d'une unique façon: celle par laquelle les dons de la nature peuvent se transformer en propriétés de l'esprit; par là, je veux dire que le génie donne forme à la matière car, en ce qui concerne la forme, l'esprit ne peut rien nommer $\operatorname{sien}^{39} »$. Il devient donc indispensable d'allier le travail au génie pour que la grâce s'inscrive dans une matière ainsi formée et appropriable.

Alors que Kant faisait de la faveur le propre d'une satisfaction désintéressée, à juste distance de l'agréable sensible et du bien moral, Schiller donne la grâce comme une faveur que la morale accorde au sensible. Même s'il replie ainsi un peu vite le bien sur le beau, Schiller retrouve la satisfaction désintéressée par son insistance sur le jeu où l'esprit et la sensibilité s'accordent plutôt que de se contraindre l'un l'autre. De façon significative, il prend l'exemple du politique pour penser ces distinctions esthétiques: à la monarchie correspond ce gouvernement de la raison sur la sensibilité et à l'ochlocratie (ou démocratie, le gouvernement par la multitude), la mainmise du sensible sur l'intelligible; mais la beauté de l'expression requiert leur accord où «s'accomplit la beauté du jeu ${ }^{40}$ ». Or, ce troisième état, cet état intermédiaire ne reçoit pas de nom politique: le gouvernement aristocratique est évoqué sans être nommé, la grâce qui lui revient devant passer aussi par un travail de chaque instant et faire de la naturalité des gestes une production culturelle, une Bildung.

Par ce travail, la «belle âme [...] contrôle les impressions humaines jusqu'à abandonner sans crainte la direction de la volonté à l'affectivité. [...] Aussi l'idée de la beauté de son comportement ne lui traverse-t-elle pas l'esprit, et il ne lui apparaît jamais qu'une autre façon d'agir et de sentir serait possible ${ }^{41} »$. À l'inverse, l'élève studieux peut toujours rendre compte de sa conformité avec la loi. La grâce consiste donc bien à avoir si impeccablement intériorisé la discipline que l'on peut sans souci s'abandonner à sa propre affectivité. Cette pathétique généralisée pousse le travail intérieur jusqu'à faire disparaître tout ce qui l'a formé, réconciliant de la sorte l'abandon et la puissance, les écarts affectifs et la centralisation du pouvoir (c'est pourquoi la femme, aux yeux de Schiller, paraît plus volontiers gracieuse en ce qu'elle s'abandonne plus naturellement).

Parallèlement à l'accord généré par la grâce couve aussi un désaccord entre la nature et l'esprit: le beau et le sublime prennent, chez Schiller, les figures de la grâce et de la dignité. La grâce tient à une liberté de mouvements dépendants de la volonté, et la dignité, à une maîtrise de mouvements indépendants de la volonté, car «dans la dignité, l'esprit règne sur

39. Ibid., p. 71, n. 5 .

4o. Ibid., p. 78.

41. Ibid., p. 83 . 
le corps et se conduit en maître [Herrscher, en souverain]; il défend son indépendance [Selbstständigkeit, son autonomie] contre les instincts despotiques [...], mais dans la grâce, au contraire, l'esprit gouverne libéralement car c'est lui qui met la nature en action ${ }^{42}$ ». Dans la dignité, la souveraineté assujettit la nature à l'esprit là où elle veut dominer (le terme employé est herrschen: c'est bien un conflit de souverainetés); dans la grâce, la nature qui se moule à ce qu'exige l'esprit, prend une apparence de liberté (einen Schein von Freywilligkeit, un aspect gracieux, spontané). Souveraineté, d'un côté; grâce, de l'autre. L'enjeu est encore plus ouvertement politique que chez Kant, avec cette nuance supplémentaire que Schiller conjoint presque immédiatement morale et dignité, liberté et libéralité. L'enjeu est aussi plus intrinsèquement social, puisque l'effet de la souveraineté peut être amorti par la grâce chez celui qui ordonne ou relevé par la dignité chez celui qui obéit: «On exige la grâce de celui qui oblige et la dignité de l'obligé. [...] Le puissant veut-il se faire aimer, qu'il devra tempérer sa supériorité de grâce. Le faible veut-il mériter l'estime, qu'il devra s'élever par la dignité au-dessus de son impuissance ${ }^{43}$.» À la différence de Kant, "la grâce schillérienne rapatrie la beauté dans le domaine de l'humanité», mais surtout dignité et grâce paraissent "constitutives de la civitas/civilitas ${ }^{44}$ », dont Éliane Escoubas signale à juste titre qu'elles forment la clef de voûte du questionnement politique de Schiller: «Le politique n'est pas par lui énoncé fondamentalement en termes de pouvoir, mais en termes de civilisation [...], en termes de culture et de formation (Bildung) ${ }^{45}$.»

\section{L'éducation esthétique de l'homme}

Cette double tournure sociopolitique est encore plus évidente dans les Lettres sur l'éducation esthétique de l'homme que Schiller compose en même temps et à la suite de La grâce et la dignité. La question que pose Schiller est simple: si l'univers physique des hommes détermine une société des forces et si le monde moral des êtres réclame une société des lois, comment peut-on passer de l'un à l'autre? L'homme de l'univers physique

42. Ibid., p. 94

43. Ibid., p. 97. Inversement, la simple apparence de dignité ou de grâce ne suffit pas lorsqu'elle ne répond pas d'un mouvement intérieur. Ce serait alors de l'affectation et «la possibilité d'observer la dignité affectée se présente aussi bien dans le cabinet du ministre que dans la salle d'étude du savant (particulièrement dans l'enseignement supérieur), autant que celle d'observer la grâce affectée au théâtre ou dans la salle de bal». Ibid., p. 105.

44. Éliane Escoubas, «La passion de la médiation: éléments pour tenter de penser le rapport à l'esthétique et du politique à partir de Friedrich Schiller», La part de l'oeil, no 12, 1996, p. 47-48.

45. Ibid., p. 44 
est bien réel et aucune idéalité morale ne peut en faire l'économie; mais l'être du monde moral est problématique, au sens où il fait problème et où il permet de poser des problèmes ${ }^{46}$. De l'un à l'autre, comme dans tout organisme vivant, on ne peut passer sans retirer de sous les pieds de l'homme-problème la réalité de l'homme-force. Un horloger peut bien arrêter le mécanisme d'une montre pour la réparer, mais la société humaine doit être réparée sans avoir à s'arrêter de vivre. Il faut donc pouvoir former l'être-problème de la morale sans abandonner la force de l'homme sensible. La solution n'est pas affaire de pouvoir (ce serait passer du côté de la force), ni de loi morale (comment s'y soumettre?). La solution réclame une éducation, la formation d'une culture (Bildung).

Pour Schiller, en chaque homme réside un «homme pur [...] représenté par l'État, lequel est la Forme objective et en quelque sorte canonique en laquelle la multiplicité des sujets aspire à se réunir ${ }^{47}$ ». Si l'État est ainsi la Forme du multiple (raison par sa forme, nature par sa multiplicité), comment l'individu pourra-t-il coïncider avec l'homme idéal qu'il contient? Soit celui-ci «étouffe l'homme empirique; l'État supprime les individus; ou bien l'individu devient État; l'homme dans le temps, en s'ennoblissant, se hausse à la stature de l'homme idéal ${ }^{48}{ }^{\prime}$. La culture est cette puissance d'anoblissement où le sensible coexiste avec le moral. Mais elle peut aisément dégénérer ou devenir plutôt contreproductive lorsque «la générosité de l'heure [der freigebige Augenblick ${ }^{49}$ " ne trouve pas de répondants. La libéralité, la grâce sont donc aussi fonction du moment historique. Comme chez Kant, il s'agit de développer une oreille qui repère les différentes tonalités de l'histoire: les instants donnés libéralement forment déjà une instance de liberté. Mais si la culture développe l'égocentrisme du goût plus que le goût de la sociabilité, alors «les classes policées [zivilisierten] nous donnent le spectacle plus repoussant encore d'un relâchement et d'une dépravation du caractère qui indignent d'autant plus qu'elles ont leur source dans la civilisation [Kultur] elle-même ${ }^{50}$ ». La culture, au lieu de conférer la liberté, redouble les besoins. En comparant les modernes Allemands aux anciens Grecs, Schiller discerne dans la division du travail toujours plus effective et dans la séparation des sciences croissante une blessure que produit la culture, dans la mesure où l'entendement moderne dissocie ce

46. "L'homme physique est une réalité, tandis que l'homme moral n'a qu'une existence problématique [problematisch].» Friedrich Schiller, Lettres sur l'éducation esthétique de l'homme (Briefe über die Æsthetische Erziehung des Menschen), trad. et préf. Robert Leroux, mise à jour par Michèle Halimi, Paris, Aubier, 1992 [1794-1795], p. 95-97.

47. Ibid., p. 103.

48. Ibid.

49. Ibid., p. 113.

50. Ibid. 
que la nature ancienne unissait. S’il est encore possible de fonder une unité, elle ne loge plus que dans la mémoire. Mais cette mémoire ressemble à une lettre morte plus qu'à une vivante intelligence. Aux besoins illusoires de la civilisation, il faudrait opposer le besoin nécessaire d'une formation de la puissance des sentiments (Ausbildung des Empfindungsvermögens).

Sur quoi baser cette formation? Dans l'expérience de l'histoire, la beauté ne semble jamais correspondre qu'à des périodes de décadence morale. Mais, à un autre niveau, la beauté répond peut-être d'un entendement plus subtil, susceptible d'alimenter une véritable culture de la cité. Pour le montrer, Schiller sent la nécessité de chercher, de la façon la plus abstraite possible, ce qui fait l'humanité de l'homme: c'est le temps, entre «quelque chose qui persiste et quelque chose qui change continuellement ${ }^{51} »$, autrement dit la personne et ses états, qui détermine deux instincts: le sensible qui insère l'homme dans les limites du temps et le transforme en matière (en remplissant ses états successifs de sensations) et le formel qui rend l'homme libre en introduisant de l'unité et de l'harmonie dans la diversité de ses manifestations (en affirmant donc la durée de sa personne). L'instinct sensible crée seulement des cas, puisqu'il est soumis au temps (il n'existe que dans le temps); l'instinct formel édicte des lois, puisqu'il épouse le temps au point de l'intérioriser (le temps est en lui).

Comme l'insociable sociabilité des hommes édifiait, pour Kant, la dynamique de la société humaine à partir même de ses contradictions, le changement persistant des êtres devrait constituer la formation d'une communauté des individus. Mais Kant et Schiller ne partagent pas le même sens de la tension: chez celui-là, la confrontation construit plutôt qu'elle ne détruit; chez celui-ci, la confrontation doit être illusoire: l'instinct sensible ne réclame du changement que dans les états; l'instinct formel n'exige de persistance que dans la personne. Leur antagonisme vient de ce que la liberté a empiété sur la vie sensible, et la puissance des sensations a pénétré dans la personnalité. «La tâche de la culture [Kultur] est de veiller sur ces deux sphères et d'assurer à chacun des instincts ses frontières ${ }^{52}$.» Ou plutôt, puisque ce sont des énergies, ils ont besoin de détentes. Un troisième instinct en fournit le principe: l'instinct de jeu qui ôte aux sensations leur puissance dynamique en les accordant aux Idées de la raison et qui enlève aux lois de la raison leur obligation morale en les conciliant avec l'intérêt des sens. Du coup, dans le jeu, l'homme apparaît comme une forme vivante (conjoignant ainsi instinct formel et instinct sensible) que Schiller nomme «beauté».

51. Ibid., p. 173 .

52. Ibid., p. 193. 
Sous l'action de la beauté, l'homme dominé par ses sentiments, tendu sensiblement, est détendu et libéré par de la forme, tandis que l'homme dominé par des lois, tendu spirituellement, est détendu et libéré par de la matière. Ainsi, de l'opposition de ces deux nécessités surgit la liberté. La disposition intermédiaire où le physique et le moral sont simultanément tendus et réciproquement détendus, Schiller la nomme l'état esthétique. Cet état est «de tous les dons, le don suprême, le don de l'humanité ${ }^{53}$ ». Mais il s'agit d'un état où l'activité mise en jeu s'appuie sur une neutralisation des agissements sensibles ou moraux; en ce sens, la beauté de l'œuvre d'art ne peut ni être passionnée, puisqu'elle doit affranchir des passions, ni devenir édifiante, puisqu'elle doit libérer de la contrainte. En décalage avec la conception rhétorique par où il devrait exister une utilité sociale du plaisir, le jeu esthétique ne doit déterminer aucun contenu sensible ou moral, mais il «rend seulement capable de ces deux activités ${ }^{54} »$. L'état esthétique se situe donc en deçà de tout investissement déterminé: il est à la fois fondamental et vide, immédiatement social et socialement sans contenu. La tâche de la culture est de fournir, en fait, aux hommes des occasions (Anlässe) ou des situations (Situationen) qui assurent la rencontre et le passage du physique au moral. La culture ne devrait donc pas être un réservoir de déterminations, mais un art des situations esthétiques ${ }^{55}$.

En faisant sentir immédiatement la forme, la beauté fait jouer de concert le sensible et le spirituel; elle les fait passer l'un en l'autre dans un présent suspendu, lui-même autonome par sa légèreté et son jeu. Comme le temps de travail était stocké dans la marchandise, le temps du jeu est préservé dans la situation esthétique. Mais, au départ, la disposition esthétique vient d'un "cadeau [Geschenk] de la nature; seule la faveur des hasards [die Gunst der Zufälle] peut délier les chaînes de l'état physique et conduire l'homme à la beauté56 ${ }^{\prime}$. La grâce répète en l'homme ce qu'il a déjà reçu de la nature. On ne doit pas s'illusionner sur ce cadeau et cette faveur. Une fois que la culture s'édifie sur l'intérêt pris aux apparences (et non seulement aux réalités matérielles des besoins physiques ou aux réalités spirituelles des nécessités morales), «l'apparence des choses est l'œuvre des hommes, et une âme qui se délecte à l'apparence prend plaisir non plus à ce qu'elle

53. Ibid., p. 279.

54. Ibid., p. 297.

55. Elle n'est pas pour autant vidée de déterminations sociales comme on le sent dans l'enthousiasme généré par la valeur nobiliaire: «D’une façon générale on appelle noble une âme qui, par la manière dont elle traite même l'occupation la plus limitée et l'objet le plus médiocre, possède le don de les transformer en un infini. On appelle noble toute forme qui imprime un caractère d'autonomie à ce qui selon sa nature ne fait que servir à une fin (n'est qu'un moyen).» Ibid., p 304-305, n. 1.

56. Ibid., p. 337. 
reçoit, mais à ce qu'elle fait ${ }^{57}$ ». C'est bien l'ordre de la production qui engendre plaisir, valeur, autonomie et jeu. La grâce et la légèreté de la disposition esthétique reposent sur l'épanouissement d'une fabrique de soi et d'une construction du monde. En se retirant de l'univers matériel de la nature, en se mettant à distance du monde réel des événements, l'homo cestheticus s'octroie un droit de propriété inaliénable sur les apparences, plus encore, sur tous les modes d'apparition (d'où son art des situations):

Comme toute existence réelle a son origine dans la nature en tant qu'elle est une force étrangère, et qu'au contraire toute apparence procède primitivement de l'homme en tant qu'il est sujet qui se représente, il ne fait qu'user de son droit absolu de propriété [absoluten Eigentumsrecht] en détachant l'apparence de l'être et en en disposant selon ses propres lois. [...] Ce droit humain de souveraineté [Herrscherrecht], il l'exerce dans l'art de l'apparence; plus il saura dans ce domaine distinguer strictement le mien du tien, séparer soigneusement l'être et la forme et donner à celle-ci autant d'autonomie que possible, plus aussi non seulement il élargira le domaine [das Reich] de la beauté, mais encore il maintiendra intactes les frontières de la vérité; car il ne saurait rendre l'apparence pure de toute réalité sans affranchir [frei zu machen, rendre libre] en même temps la réalité de l'apparence ${ }^{58}$.

Le vocabulaire politique et juridique indique assez que cet empire des apparences n'est pas coupé de toute effectivité sociale. Le jeu des apparences, loin de ruiner le principe de réalité, lui donne, selon Schiller, un éclat particulier, où le moral peut légitimement écrire ses lois sur le sensible sans que celui-ci en sente les contraintes, puisque le détachement des apparences aura permis d'en détendre les résistances et d'en étendre la liberté.

La culture rend ainsi possible la construction d'une société où les royaumes autonomes du sensible et du spirituel sont à la fois respectés et intégrés l'un en l'autre. Là où règnent le goût des apparences et l'autonomie du jeu, «l'idéal gouverne la vie, l'honneur triomphe sur le désir des possessions, la pensée sur la jouissance, le rêve de l'immortalité sur l'attachement à l'existence. La voix publique y sera seule redouté ${ }^{59}{ }$. Ce sont encore tous les dispositifs mémoriels classiques qui investissent l'idéal de l'existence, mais en passant par l'apparence de l'opinion publique à produire plutôt que par l'héritage d'une communauté de vérités à transmettre. Au moment où la vie triomphe, jusqu'à la mise en place d'une biopolitique, comme la nomme Michel Foucault, cette gestion sociale des existences doit passer par un fondement esthétique qui, délibérément, se situe en retrait des réalités afin de mieux assurer leur maîtrise culturelle. «En retrait des

57. Ibid., p. 339 .

58. Ibid., p. 343 .

59. Ibid., p. 347 . 
réalités» ne signifie pas que l'esthétique prendrait pied sur des nuages par définition volatils et évanescents, mais qu'elle fait fond sur des effets retirés du commerce des marchandises, sur du travail impeccablement improductif, sur une mémoire et une grâce que la fabrication du moi social semble, pourtant, avoir déjà lourdement empâtées. Schiller prend encore pour exemple de cet art des situations et des dispositifs esthétiques la rhétorique sociale de la politesse : celui qui lit dans les protestations d'amitié une forme de sympathie personnelle manque du sens de l'autonomie des apparences; celui qui appelle la dissimulation à son aide pour flatter et plaire manque du principe de réalité. Schiller retraduit ainsi dans le creux de l'esthétique moderne les usages anciens de l'honnêteté mondaine.

La grâce et le don ouvrent sur la liberté parce qu'ils ont été retirés du cadre traditionnel de l'obligation et de la réciprocité. Dans le monde des marchandises et de la production, ils figurent comme des valences libres dont on peut exploiter le jeu devenu apparemment autonome. Il suffit de souscrire au renversement des réalités sociales telles qu'elles sont conçues: si l'univers des hommes est régi par la force et le souci de se conserver, si les individus sont au départ isolés et antagonistes, alors la politesse, le jeu des apparences, la grâce, la faiblesse deviennent des enjeux politiques par où il paraît possible de bâtir une société de liberté: «Le besoin de plaire soumet l'homme fort à la juridiction délicate du goût; il peut dérober la volupté, mais l'amour doit être un don. [...] Désormais la faiblesse devient sacrée et la force qui ne se maîtrise pas déshonore; on corrige l'injustice de la nature par la générosité de mœurs chevaleresques ${ }^{60}$.» Ainsi, le besoin contraint l'individu à constituer une société, la raison lui inculque des principes de sociabilité, mais la disposition esthétique seule lui alloue un caractère sociable: la culture est ce qui permet d'intérioriser la sociabilité, de faire de l'intersubjectif la constitution de sa propre subjectivité.

Loin de retirer le monde de l'art et du beau des pratiques sociales, la culture esthétique a pour tâche de redonner aux individus le sentiment immédiat qu'ils sont des êtres sociaux, qu'ils partagent une même communauté. L'art dans la culture et la culture dans la société font des apparences leur royaume afin de mieux assurer la prise réelle que les hommes auraient sur leur destin, afin de mieux jouer dans les effets d'immédiateté les aveuglantes médiations sociales. Le retrait ou la mise à l'écart de l'activité esthétique au sein des pratiques quotidiennes (jusqu'à sa sacralisation ou à son mépris) ne sont eux-mêmes que des apparences, mais des apparences nécessaires.

6o. Ibid., p. 365 . 